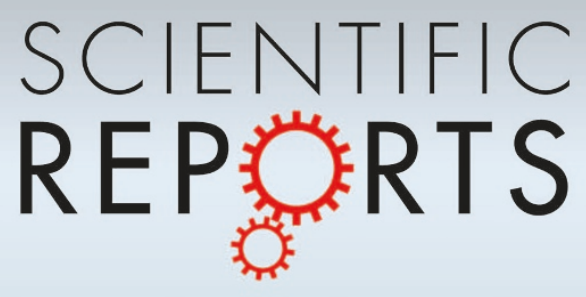

\title{
OPEN A Highly Reversible Lithium Metal Anode
}

SUBJECT AREAS:

BATTERIES

CHEMICAL PHYSICS

STATISTICAL MECHANICS

COMPUTATIONAL CHEMISTRY

Received

12 June 2013

Accepted

2 January 2014

Published

22 January 2014

Correspondence and requests for materials should be addressed to M.S.P. (ms91.park@ samsung.com) or D.I.

(dongmin.im@ samsung.com)

* These authors contributed equally to this work.

\author{
Min Sik Park ${ }^{1 *}$, Sang Bok Ma ${ }^{*}$, Dong Joon Lee', Dongmin Im', Seok-Gwang Doo' \& Osamu Yamamoto²
}

'Energy Lab., Samsung Advanced Institute of Technology, Samsung Electronics, 130 Samsung-ro, Suwon, Gyeonggi-do 443-803, Republic of Korea, ${ }^{2}$ Department of Chemistry, Mie University, Tsu 51 4-8507, Japan.

Lithium metal has shown a lot of promise for use as an anode material in rechargeable batteries owing to its high theoretical capacity. However, it does not meet the cycle life and safety requirements of rechargeable batteries owing to electrolyte decomposition and dendrite formation on the surfaces of the lithium anodes during electrochemical cycling. Here, we propose a novel electrolyte system that is relatively stable against lithium metal and mitigates dendritic growth. Systematic design methods that combined simulations, model-based experiments, and in situ analyses were employed to design the system. The reduction potential of the solvent, the size of the salt anions, and the viscosity of the electrolyte were found to be critical parameters determining the rate of dendritic growth. A lithium metal anode in contact with the designed electrolyte exhibited remarkable cyclability (more than $\mathbf{1 0 0}$ cycles) at a high areal capacity of $12 \mathrm{mAh} \mathrm{cm}$.

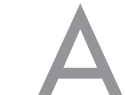

nodes of lithium metal are being considered in the development of rechargeable batteries with high energy densities ${ }^{1,2}$. The recent escalation in the demand for long-range electric vehicles (EVs) has rekindled research on lithium-air and lithium-sulfur batteries in which Li metal anodes are an essential component ${ }^{3-6}$. While Li metal has a theoretical capacity $\left(3860 \mathrm{mAh} \mathrm{g}^{-1}\right)$ that is ten times larger than that of the graphite anodes $\left(372 \mathrm{mAh} \mathrm{g}^{-1}\right)$ used in Li-ion batteries, another important requisite for fabricating cells with high energy densities is that the capacity per unit electrode area (areal capacity of the electrodes) should also be high. Figure 1 illustrates the dependence of cell energy density on areal capacity for Li-ion, Li-S, and Li-air batteries (see Supplementary Table S1 for the assumptions related to and details of the calculations), where the energy density of a Li-ion battery is almost saturated after $6 \mathrm{mAh} \mathrm{cm}^{-2}$. On the contrary, the energy densities of $\mathrm{Li}-\mathrm{S}$ and $\mathrm{Li}$-air batteries increase without saturation. It is obvious that $\mathrm{Li}-\mathrm{S}$ and Li-air batteries have the potential to considerably improve the driving ranges of EVs. However, longrange driving is feasible only when lithium anodes with significantly large areal capacities, preferably $10 \mathrm{mAh}$ $\mathrm{cm}^{-2}$ or higher, are used.

In rechargeable lithium batteries, an areal capacity of $10 \mathrm{mAh} \mathrm{cm}^{-2}$ corresponds to the repeated stripping and plating of a lithium layer that measures about $50 \mu \mathrm{m}$ in thickness. This large change in the volume of the $\mathrm{Li}$ electrode can cause two serious problems: (1) continuous decomposition of the electrolyte owing to contact with the fresh Li surface through cracks in the solid-electrolyte interphase $(\mathrm{SEI})^{7} ;$; 2 acceleration of dendritic growth because of the presence of spatially inhomogeneous SEIs and non-uniform morphology of the Li surface $e^{8-16}$. These two factors can seriously affect the performance, cycle life, and safety of lithium batteries. Hence, the prevention of electrolyte decomposition and the suppression of dendritic growth are necessary if Li anodes are to be employed in batteries. It had been previously suggested that the surface morphologies of lithium anodes could be controlled through the use of various electrolytes such as carbonates, esters, ethers, and ionic liquids, or mixtures of such electrolytes ${ }^{8-17}$. Furthermore, exploiting the pressure effect ${ }^{18}$, using thin films of lithium ${ }^{19}$, and applying block copolymer electrolytes ${ }^{20}$ have also been suggested as ways of enabling efficient cycling at low areal capacities.

In our study, we systematically examined the effect of electrolyte composition on the cycling of Li anodes by combining multiple methods. We screened electrochemically stable liquid solvents against Li metal anodes using density functional theory (DFT) calculations and investigated the interactions between these screened solvents and the Li surfaces using ab initio molecular dynamics (AIMD) simulations. Additionally, we conducted experiments using Li symmetric cells to identify the key parameters affecting the dendrites' growth rate. To further analyze the impact of these key parameters, in situ optical microscopy was performed and a statistical model of the parameters was investigated. Finally, Li symmetric cells were prepared using the designed electrolytes and cycled at an areal capacity of $12 \mathrm{mAh} \mathrm{cm}^{-2}$. To our knowledge, this is the first instance of cycling tests being performed under such severe conditions. 


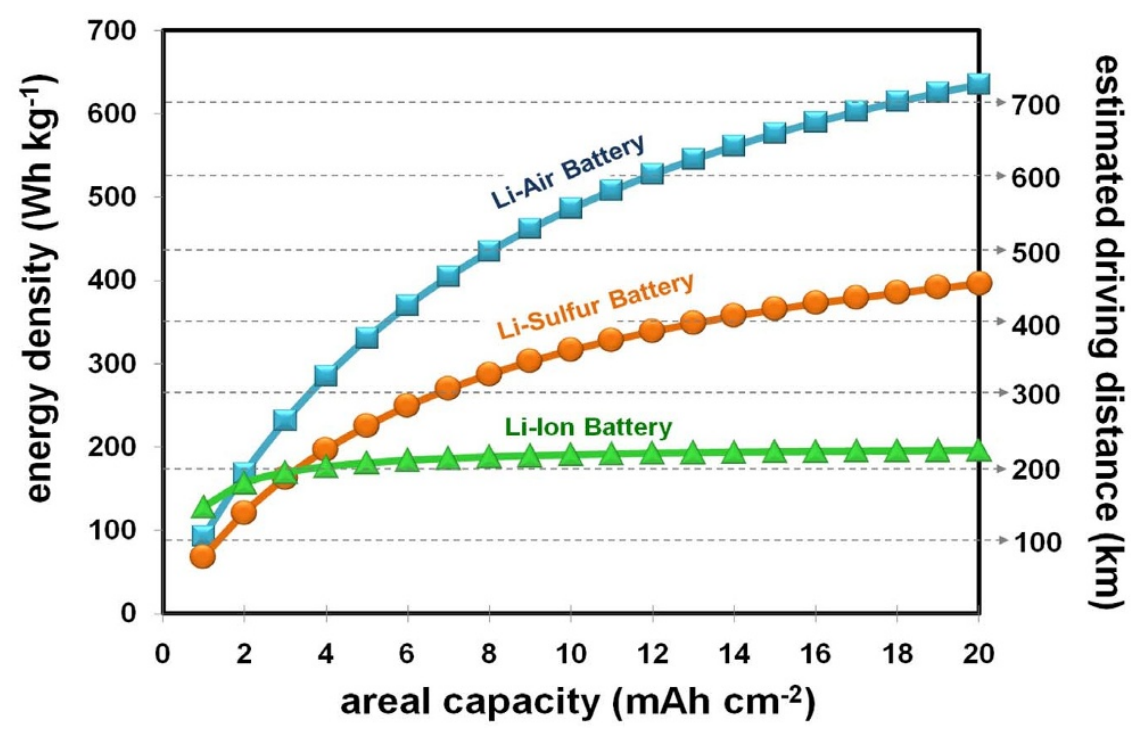

Figure $1 \mid$ Dependence of the energy density of a battery cell on the areal capacity of the electrode for $\mathrm{Li}$-air, $\mathrm{Li}-\mathrm{S}$, and $\mathrm{Li}$-ion batteries, and the estimated driving distance of an electric vehicle with respect to the energy density of the battery cell used. The energy densities of the battery cells were calculated assuming that they all had the same cell structure, namely, one comprising a "current collector-cathode-electrolyte-separator-protective layer-anode-current collector", with no cell packing components used. The driving distances were estimated on the basis of the car Nissan Leaf, which uses Li-ion battery cells ${ }^{3}$ with an energy density of $140 \mathrm{Wh} \mathrm{kg}^{-1}$ and has a driving range of $160 \mathrm{~km}$ (for details, see Supplementary Table S1).

\section{Results}

The first step was to screen various solvents on the basis of their stability against lithium metal. Three groups of solvents ${ }^{21}$ were considered as candidates (see Supplementary Table S2), and the reduction potential of each solvent molecule was calculated using a method based on $a b$ initio DFT. Table 1 lists 22 solvents whose calculated reduction potentials against $\mathrm{Li}^{+} / \mathrm{Li}$ are less than $-0.5 \mathrm{~V}\left(\right.$ vs. $\left.\mathrm{Li}^{+} / \mathrm{Li}\right)$, implying that the probability of a direct reaction between these solvents and lithium metal would be low. While solvent-salt interactions were not considered for these calculations, the criterion of a reduction potential below $-0.5 \mathrm{~V}$ (vs. $\mathrm{Li}^{+} / \mathrm{Li}$ ) was thought to be adequate for screening purposes, since a previous study ${ }^{22}$ had suggested the energy levels in glymes were lowered by $0.5 \mathrm{eV}$ on average when the solvent-salt interactions were considered. The screened solvents included six linear ethers, five cyclic ethers, two aromatic ethers, two carbonates, two amides, an amine, and a urea (see Table 1). Except for the amide and the urea, all the screened solvents had a dielectric constant ${ }^{21}$ of less than 10; of the linear ethers considered, the shorter ones exhibited lower reduction potentials; the cyclic ethers showed lower reduction potentials than did the aromatic ones with conjugated rings. Therefore, it was inferred that the criteria of uniform charge distribution (low dielectric constant), short chain length (among linear ethers), and non-conjugated rings (among cyclic ethers) might aid in the design of solvent molecules with better electrochemical stability against reduction. Based on these computationally screened solvents, we performed experiments on the solubility of Li salt for further screening. As shown in Supplementary Table S3, 11 solvents exhibiting solubilities of more than 1.0 M for lithium tetrafluoroborate $\left(\mathrm{LiBF}_{4}\right)$ salt were screened for the following experiments.

To identify the critical parameters affecting the growth of dendrites, we considered the properties of Li metal, as well as properties of interfaces between Li metal and the electrolytes. We have already shown in our previous work ${ }^{23}$ that the intrinsic properties of $\mathrm{Li}$ surfaces do not significantly affect the formation of dendrites. Hence, we shifted our focus to the interfaces formed between $\mathrm{Li}$ surfaces and the electrolytes investigated. Accordingly, the effects of four parameters, namely, the viscosities of the bulk electrolytes $(\eta)$, the interfacial energies between Li metal and the solvents $(\gamma)$, the concentrations of $\mathrm{Li}$ ions $\left(C_{\mathrm{Li}+}\right)$ in the electrolytes, and the size of the salt anions $\left(R_{\mathrm{A}}\right)$ were investigated in detail. Some of these parameters have been studied by Monroe and Newman ${ }^{24}$ using a phenomenological model. We measured the short-circuit time $\left(t_{\mathrm{s}}\right)$ of Li symmetric coin cells (Fig. 2a) as an indicator of the dendrites' growth rate. The compositions of the electrolytes were chosen such that they bore representative values of the parameters $\eta, \gamma, C_{\mathrm{Li}+}$, and $R_{\mathrm{A}}$, with the solvents being some of the screened ones mentioned above (see Table 2 for the sets of electrolytes, where seven solvents-1,2dimethoxyethane (DME); tetraglyme; polyglyme; tetrahydrofuran (THF); 2-methyl THF; 2,2-dimethyl THF; 2,5-dimethyl THF-were selected from the 11 screened solvents in Supplementary Table S3).

The viscosity of the electrolyte used in a battery may influence the movement of Li ions and affect dendritic growth. To study the effect of $\eta$, we chose linear ethers for the solvents since the values of $\eta$ could be controlled by varying the molecular lengths of the ethers without significantly affecting the other parameters. It is known that differences in the molecular weights of solvents usually lead to differences in their viscosities as well. Therefore, the $t_{\mathrm{s}}$ values of Li symmetric cells that used $1 \mathrm{M}$ lithium bis(trifluoromethanesulfonyl)imide (LiTFSI) in DME $\left(90.12 \mathrm{~g} \mathrm{~mol}^{-1}\right)$, tetraglyme $\left(222.28 \mathrm{~g} \mathrm{~mol}^{-1}\right)$, and polyglyme $\left(\sim 500 \mathrm{~g} \mathrm{~mol}^{-1}\right)$ were measured. Figure $2 \mathrm{~b}$ shows the lithium deposition profiles of these Li symmetric cells at a current density of $1 \mathrm{~mA} \mathrm{~cm}^{-2}$ for the selected electrolytes. The Li symmetric cell using DME showed the highest $t_{\mathrm{s}}(\sim 74 \mathrm{~h})$ and the lowest overpotential $(<0.07 \mathrm{~V})$ during lithium deposition, indicating that DME was the solvent that was most effective in reducing dendritic growth. The average $t_{\mathrm{s}}$ values of the cell using tetraglyme was $23 \mathrm{~h}$, which was smaller than that of the DME-containing cell ( $56 \mathrm{~h})$, indicating faster dendritic growth. The overpotential of the tetraglyme-containing cell was also higher than that of the DME-containing cell. The potential of the Li symmetric cell using polyglyme jumped dramatically to over $2.5 \mathrm{~V}$ right after the start of lithium deposition and then increased gradually to $5 \mathrm{~V}$, suggesting that the resistance of the polyglymecontaining cell was so high that lithium could not be deposited readily.

Higher interfacial energy between Li metal and the solvent molecules may retard dendrite formation since they would result in larger surface areas. By analyzing the results of the AIMD simulations 
Table 1 | Reduction potentials of the screened solvents. The screened solvents had reduction potentials below $-0.5 \mathrm{~V}$ (for the full list of the candidate solvents, see Supplementary Table S2). The red, blue, and brown letters correspond to low-permittivity electron donors, aprotic protophobic, and aprotic protophilic solvents, respectively

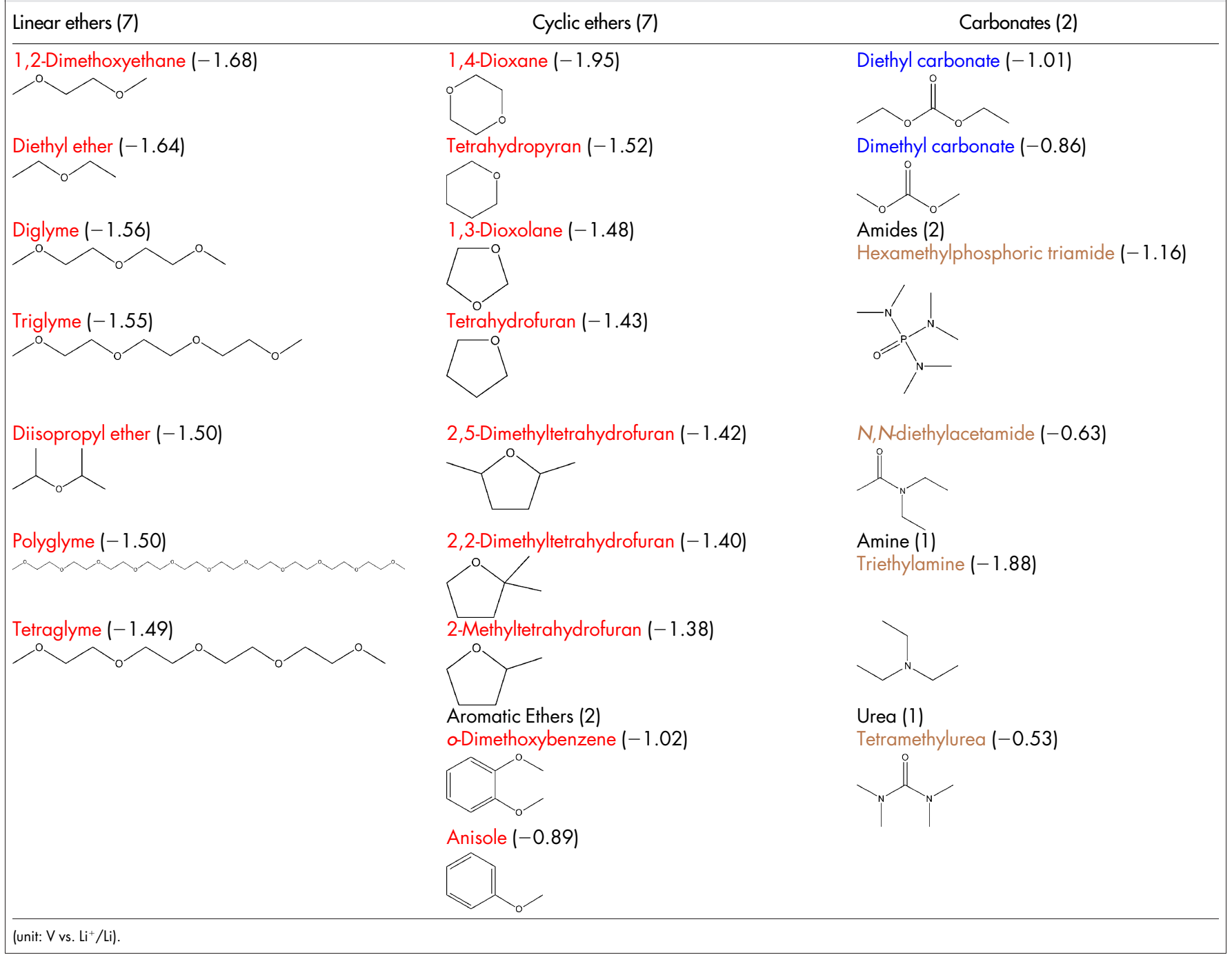

performed for the screened solvent molecules listed in Table 1, we found that the oxygen atoms in the ether molecules are attracted to the Li metal surface (Supplementary Fig. S1). Therefore, in order to control the value of $\gamma$, we selected four of the screened cyclic ethers: THF; 2-methyl THF; 2,2-dimethyl THF; and 2,5-dimethyl THF. It was assumed that the existence of methyl group(s) in the molecules of 2-methyl THF; 2,2-dimethyl THF; and 2,5-dimethyl THF would increase $\gamma$ owing to a steric effect. Indeed, the values of $\gamma$ calculated for THF; 2-methyl THF; 2,2-dimethyl THF; and 2,5-dimethyl THF via the AIMD simulations were $0.453,0.506,0.526$, and $0.567 \mathrm{~J} \mathrm{~m}^{-2}$, respectively (Supplementary Fig. S2), confirming the assumption regarding the steric effect. Consequently, the effect of $\gamma$ on dendritic growth was investigated by measuring the values of $t_{\mathrm{s}}$ of Li symmetric cells that used $1 \mathrm{M}$ LiTFSI in THF; 2-methyl THF; 2,2-dimethyl THF; and 2,5-dimethyl THF. While the overpotential of the Li symmetric cells increased with the number of methyl groups present in the solvent, the differences in the $t_{\mathrm{s}}$ values were negligible, judging from the profiles of the cells. The average $t_{\mathrm{s}}$ values were $40,44,45$, and $44 \mathrm{~h}$, for the Li symmetric cells employing THF; 2-methyl THF; 2,2dimethyl THF; and 2,5-dimethyl THF; respectively (Table 2). The $t_{\mathrm{s}}$ values increased slightly in the case of the solvents that contained methyl groups. However, it was revealed that the change in $\gamma$ induced by each solvent was not a dominant factor in determining $t_{\mathrm{s}}$.
It was assumed that a higher $C_{\mathrm{Li}}$, would increase the number of $\mathrm{Li}$ ions present near the Li anode surface, resulting in spatially uniform Li plating. To investigate the effect of an increase in $C_{\mathrm{Li}}+$ of the electrolyte on dendritic growth, lithium deposition was allowed to occur in Li symmetric cells using $0.5,1.0,2.0$, and 3.0 M LiTFSI in tetraglyme. The average $t_{\mathrm{s}}$ decreased slightly with an increase in $C_{\mathrm{Li}+}$ to up to $2.0 \mathrm{M}$ (Table 2), implying that the increase in $C_{\mathrm{Li}}+$ could not mitigate dendritic growth. Instead, it is more plausible that the increase in concentration might have increased the viscosity of the electrolyte, thus accelerating dendritic growth to some degree. In the case where 3.0 M LiTFSI in tetraglyme was used, the potential of the Li symmetric cell jumped to $1.25 \mathrm{~V}$ immediately and then steadily increased to $4 \mathrm{~V}$. The resistance of the 3.0 M LiTFSI cell was so high that measuring $t_{\mathrm{s}}$ was not possible.

A larger $R_{\mathrm{A}}$ may have increased the degree to which Li ions contributed to the ionic conductivity of the cells, since the movement of the anions might be retarded owing to their large size. In other words, a larger $R_{\mathrm{A}}$ might increase the transference number of lithium ions ${ }^{25}$. In order to investigate the effect of anion size on dendritic growth, we next selected three different salts whose anions had distinct radii to form the electrolyte: lithium iodide (LiI), $\mathrm{LiBF}_{4}$, and LiTFSI. The radii of the anions $\mathrm{I}^{-}, \mathrm{BF}_{4}^{-}$, and $\mathrm{TFSI}^{-}$were estimated to be $0.206,0.24$, and $0.326 \mathrm{~nm}$, respectively $\mathrm{y}^{26,27}$. Therefore, the average $t_{\mathrm{s}}$ values of $\mathrm{Li}$ 

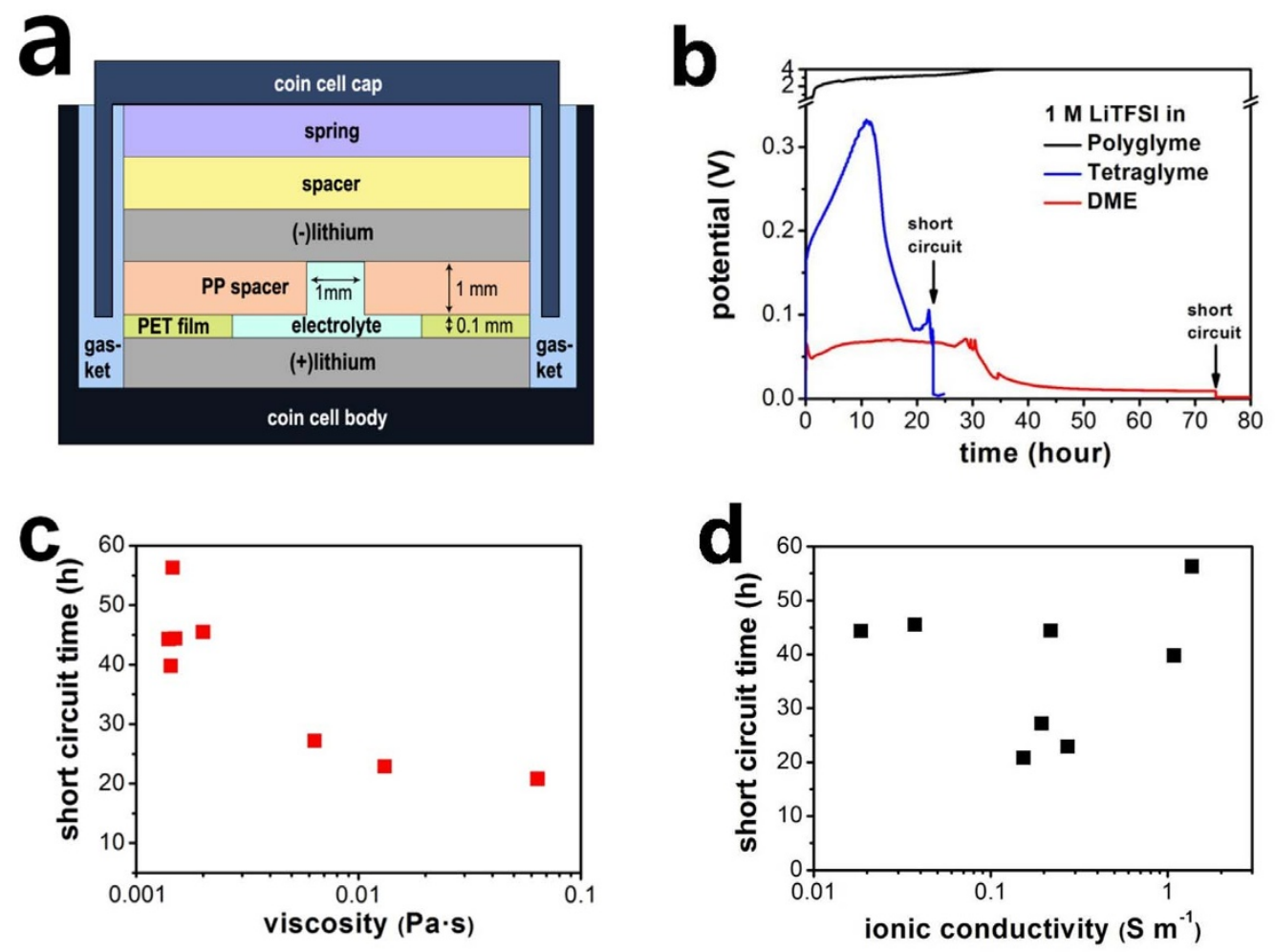

Figure $2 \mid$ Measurement of the short-circuit time. (a), The Li symmetric cells used to evaluate the effect of the choice of liquid electrolyte on the growth of lithium dendrites. (b), Lithium deposition profiles of the Li symmetric cells for a current density of $1 \mathrm{~mA} \mathrm{~cm}^{-2}$, showing the effect of the molecular weight of the solvent used. The dependence of $t_{\mathrm{s}}$ of the Li symmetric cells on (c), the viscosities and (d), the ionic conductivities of various screened solvents. The same salt (LiTFSI) was used with all the solvents.

symmetric cells using $1 \mathrm{M} \mathrm{LiI,} \mathrm{LiBF}_{4}$, and LiTFSI in tetraglyme were measured and found to be 7,14 , and $23 \mathrm{~h}$, respectively (Table 2 ). The value of $t_{\mathrm{s}}$ for the Li symmetric cell with LiTFSI was the highest, while the overpotential of the Li symmetric cell with LiI was the highest.

To further investigate the relationship between $t_{\mathrm{s}}$ and the kinetic properties of the electrolytes, the viscosities and ionic conductivities of all the electrolytes mentioned above were measured (Table 2). Figures $2 \mathrm{c}$ and $2 \mathrm{~d}$ show the dependence of the $t_{\mathrm{s}}$ values of the $\mathrm{Li}$ symmetric cells on the viscosities and ionic conductivities, respectively, of the eight electrolytes formed using the salt LiTFSI (i.e., DME; THF; 2-methyl THF; 2,2-dimethyl THF; 2,5-dimethyl THF combined with $1 \mathrm{M}$ LiTFSI; and TEGDME combined with 0.5, 1.0, 2.0 M LiTFSIs). The data for the other salts have been excluded to avoid the effect of their anions' sizes. It is obvious that their values of $t_{\mathrm{s}}$ were inversely proportional to the viscosity of the electrolyte, regardless of the other electrolyte-related parameters such as $\gamma$ and $C_{\mathrm{Li}+}$ (Fig. 2c). This confirms again that the viscosity of the electrolyte was one of the critical factors determining the dendrites' growth rate. However, a

Table 2 | Viscosities and ionic conductivities of selected screened electrolytes such that they covered a range of the four parameters investigated; values of short-circuit time $\left(t_{s}\right)$ of Li symmetric cells containing these electrolytes. To ensure the reliability of the $t_{\mathrm{s}}$ data, several cells were tested for each case and the $t_{\mathrm{s}}$ values of the cells were then averaged

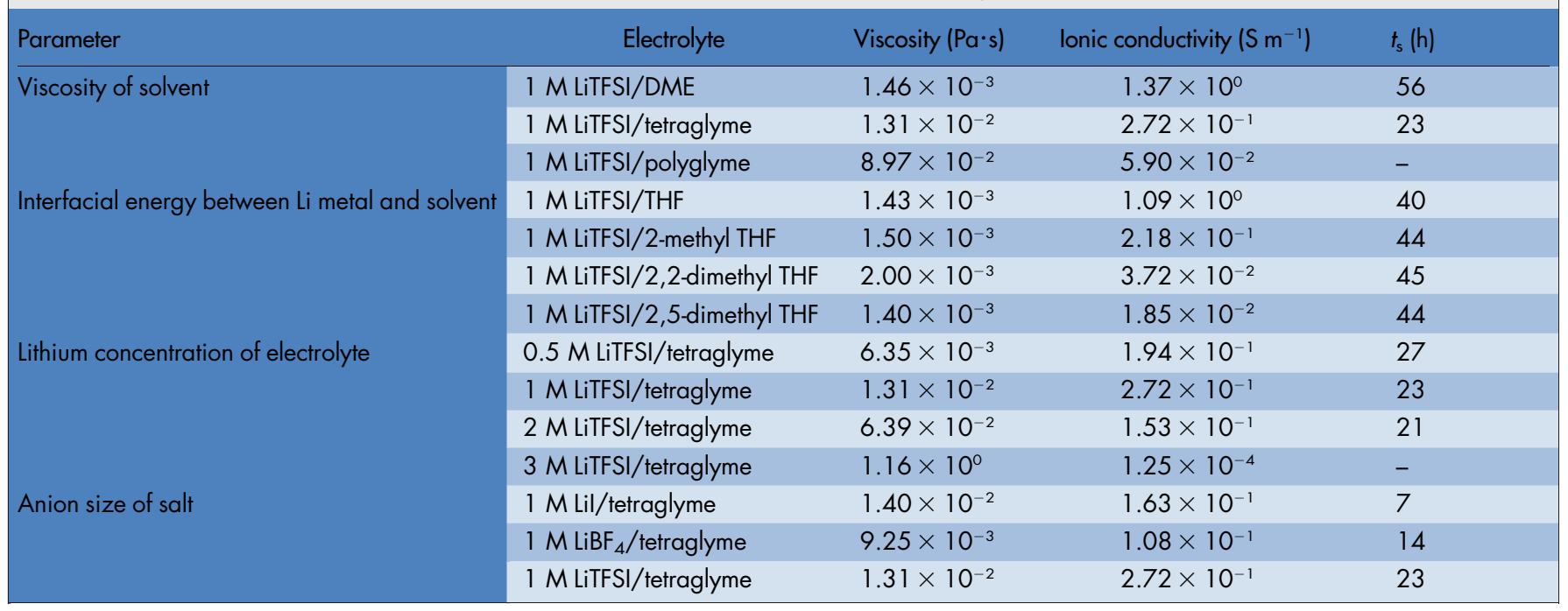



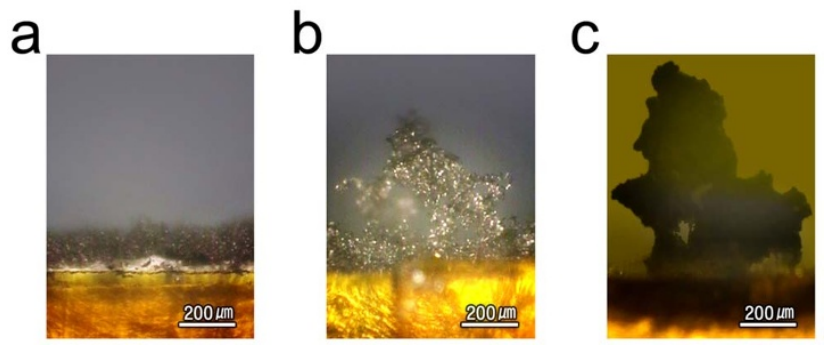

Figure 3 Images of lithium dendrites taken by a microscope during the in situ observation of lithium deposition. The electrolytes used were a, (a) 1 M solution of LiTFSI in DME, (b), a 1 M solution of LiTFSI in tetraglyme, and (c), a $1 \mathrm{M}$ solution of LiI in tetraglyme at an areal capacity of $1.67 \mathrm{mAh} \mathrm{cm}^{-2}$.

direct relationship between $t_{\mathrm{s}}$ and the ionic conductivity of the electrolytes was not found, as can be seen in Fig. 2 d. This is demonstrated particularly clearly by the set of electrolytes selected for the experiment based on the range of $\gamma$ values (Table 2). For a significant range of the ionic conductivity between 1.09 and $0.0185 \mathrm{~S} \mathrm{~m}^{-1}$, there was no change in the $t_{\mathrm{s}}$ values for an almost constant viscosity, clearly indicating that dendritic growth was independent of the ionic conductivity of the electrolyte. To summarize the results of the measurements of the $t_{\mathrm{s}}$ values, the viscosity of the electrolyte and the size of its anion were found to be the factors with the most influence on dendritic growth.

\section{Discussion}

In order to directly observe the effects of the viscosity and anion size on dendritic growth, an electrochemical cell was set up such that it could be observed using in situ optical microscopy (Supplementary Fig. S3). The dendritic growth on the Li electrode surface was recorded in real time during lithium deposition using a $1 \mathrm{M}$ solution of LiTFSI in DME (T-DME), a $1 \mathrm{M}$ solution of LiTFSI in tetraglyme (T-tetraglyme), and a $1 \mathrm{M}$ solution of LiI in tetraglyme (I-tetraglyme) at a current density of $5 \mathrm{~mA} \mathrm{~cm}^{-2}$. A comparison of the cells using T-DME and T-tetraglyme elucidated the effect of electrolyte viscosity on dendritic growth, while those using T-tetraglyme and Itetraglyme did the same for the effect of anion size. Figure 3 shows the morphologies of the deposited lithium layers when T-DME, Ttetraglyme, and I-tetraglyme were used. The micrographs were taken after a charge of $1.67 \mathrm{mAh} \mathrm{cm}^{-2}$ had passed for each experiment. The thicknesses of the deposited lithium layers were 130, 570, and $800 \mu \mathrm{m}$ for T-DME, T-tetraglyme, and I-tetraglyme, respectively. Since the thickness of each deposited lithium layers should

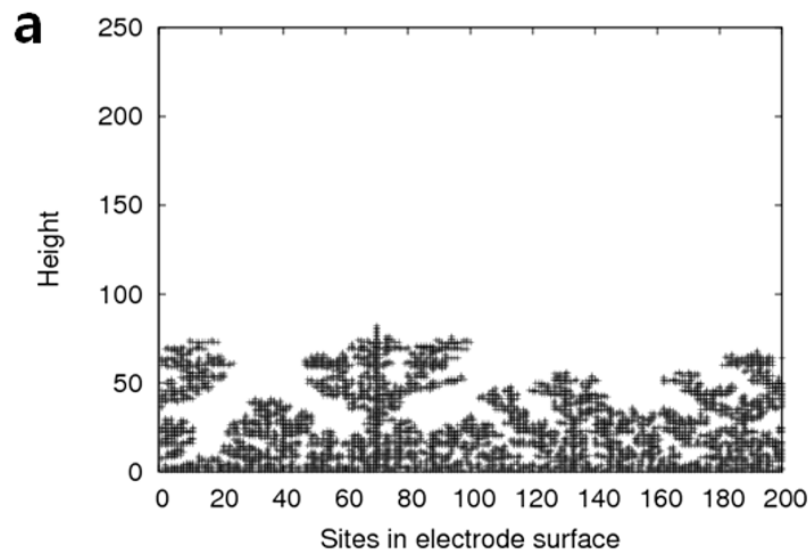

theoretically have been as low as $8 \mu \mathrm{m}$, the results indicate that lithium was deposited in the form of dendrites in all cases. It was also reconfirmed that DME was more effective than tetraglyme as a solvent for mitigating dendritic growth, owing to the lower viscosity of the former and higher effectiveness of LiTFSI in generating dense lithium deposits than LiI because the $\mathrm{TFSI}^{-}$anions are larger in size.

To study the effect of $\eta$ in detail, we performed statistical simulations using two-dimensional diffusion-limited aggregation (DLA) models ${ }^{28,29}$. Figure 4 shows the DLA simulation results for two different mean collision times $(\tau)$, with the value of $\tau$ for the model in Fig. 4a being nine times greater than that for the model in Fig. $4 \mathrm{~b} . \tau$, which is defined as the average time between collisions of particles in random motion, is proportional to the mobility $(u)$, as shown by the equation $^{25,30}$

$$
u=v_{\mathrm{d}} / F=\tau / m
$$

where $v_{\mathrm{d}}, F$, and $m$ are the drift velocity of the particles, the external force to which the particles are subjected, and the mass of an individual particle, respectively. According to the Stokes-Einstein equation, a particle randomly moving in a viscous medium experiences a drag force $\left(F_{\mathrm{d}}\right)$ exerted by its environment, i.e., the medium. Hence,

$$
F_{\mathrm{d}}=-F=-6 \pi r \eta v_{\mathrm{d}}
$$

where $r$ is the radius of the particle. By combining equations (1) and (2), we obtain

$$
u=v_{\mathrm{d}} /\left(-F_{\mathrm{d}}\right)=\tau / m=1 /(6 \pi r \eta)
$$

Therefore, the DLA simulation for which the value of $\tau$ was nine times greater corresponded to the motion of Li ions in a solvent whose viscosity was nine times lower. The results of the DLA simulations, shown in Fig. 4, were comparable to those of the in situ observations, which are shown in Fig. 3, with the viscosity corresponding to the results in Fig. 3a being nine times lower than that for the results in Fig. 3b (Table 2). The fact that $\tau$ for the simulation in Fig. 4a was higher suggests dendritic growth with a dense and thick morphology and one that is similar to the growth shown in Fig. 3a. Similarly, the smaller $\tau$ for the simulation in Fig. $4 \mathrm{~b}$ indicates a long and thin morphology similar to that seen in Fig. 3b. When the value of $\tau$ of the Li ions near the tops of the dendrites was high, the probability of the ions reaching sites within the dendrites increased, resulting in densification of the dendrites.

To check whether the design principles drawn from the results of this study were valid, coin cells were prepared and tested. These cells contained two lithium electrodes facing each other; DME (low

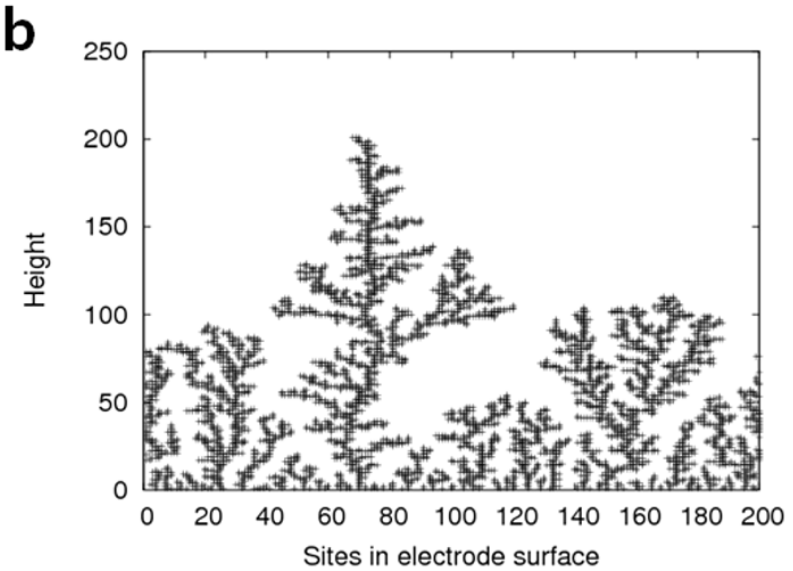

Figure 4 Statistical DLA model for the simulation of dendritic growth. (a), (b), The mean collision time for Li ions for (a) was nine times greater than that for (b). (a) and (b) correspond to Figs. $3 \mathrm{a}$ and 3b, respectively, with the viscosity related to Fig. $3 \mathrm{~b}$ being nine times greater than that for Fig. $3 \mathrm{a}$. It was determined from equation (3) that the mean collision time of Li ions was inversely proportional to the viscosity of the electrolyte used. 


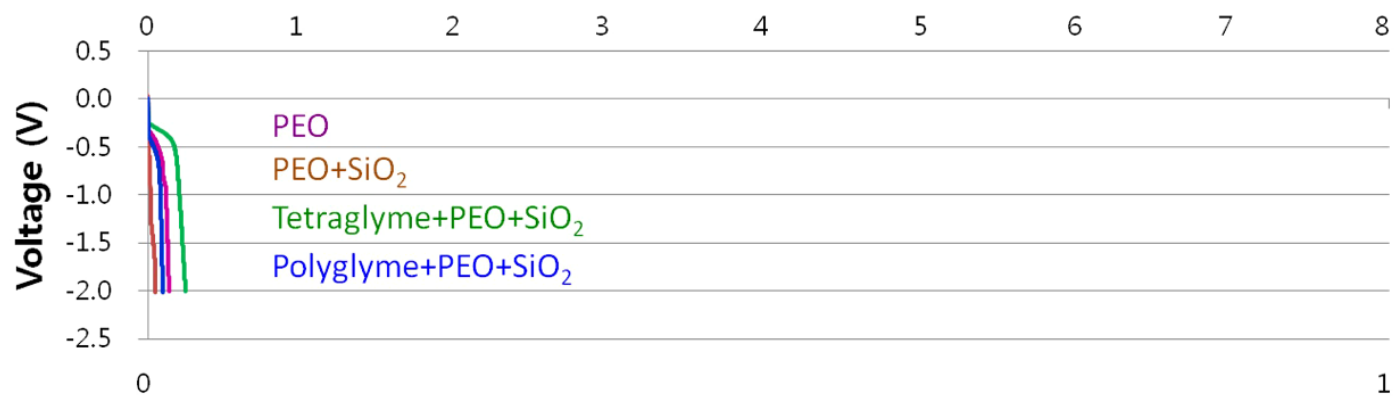

\section{Cycle number}
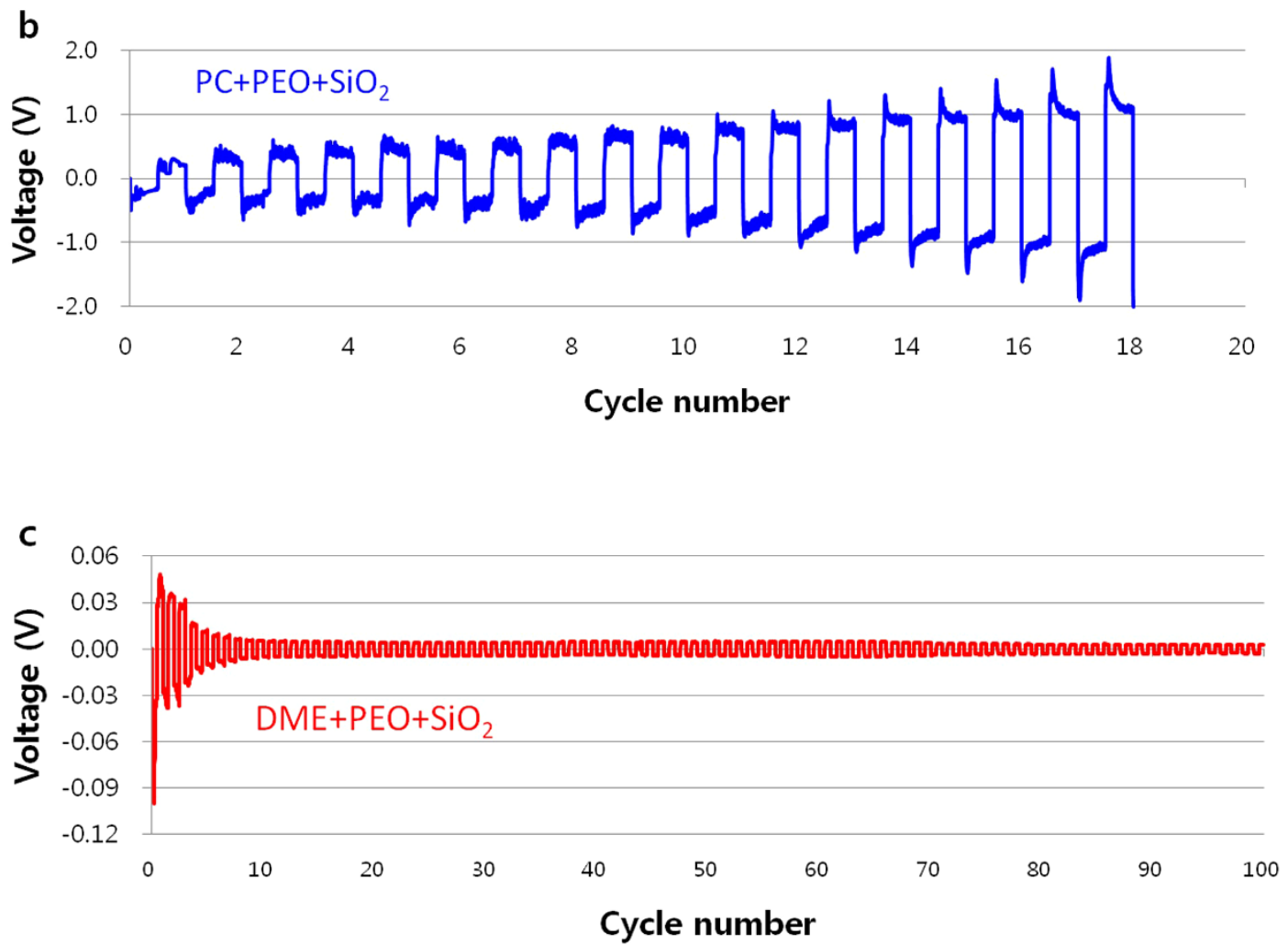

Figure 5 Cycling profiles of $\mathrm{Li}$ symmetric cells that used different electrolytes. The electrolytes used were (a), $\mathrm{PEO}, \mathrm{PEO}+\mathrm{SiO}{ }_{2}$, tetraglyme $+\mathrm{PEO}+$ $\mathrm{SiO}_{2}$, and polyglyme $+\mathrm{PEO}+\mathrm{SiO}_{2}$, (b), $\mathrm{PC}+\mathrm{PEO}+\mathrm{SiO}_{2}$, and (c), DME $+\mathrm{PEO}+\mathrm{SiO}_{2}$. The same salt (LiTFSI) was used in all of the cells.

reduction potential and low $\eta$ ) and LiTFSI (large $R_{\mathrm{A}}$ ) were chosen as the representative solvent and salt, respectively, for the cells. We also combined DME and LiTFSI with a polyethylene oxide (PEO)-based electrolyte that contained silica as a filler and reinforced by a commercial polypropylene separator to further mitigate dendritic growth. Although the ionic conductivity of PEO is low, it is stable with respect to $\mathrm{Li}$ metal and has the ability to suppress directional dendritic growth. It is also known that a $\mathrm{SiO}_{2}$ filler increases the dissociation of Li salts ${ }^{31-35}$ and enhances the mobility of $\mathrm{Li}$ ions ${ }^{1}$ in polymer electrolytes. In addition, compressive pressure was applied using a coil spring installed in the coin cell ${ }^{18}$. For comparison, cells that used $\mathrm{PEO}, \mathrm{PEO}+\mathrm{SiO}_{2}$, tetraglyme $+\mathrm{PEO}+\mathrm{SiO}_{2}$, polyglyme $+\mathrm{PEO}+\mathrm{SiO}_{2}, \mathrm{DME}+\mathrm{PEO}+\mathrm{SiO}_{2}$, and propylene carbonate (PC) $+\mathrm{PEO}+\mathrm{SiO}_{2}$ as electrolytes were assembled and cycled at a current density of $3 \mathrm{~mA} \mathrm{~cm}{ }^{-2}$ and capacity of $12 \mathrm{mAh} \mathrm{cm}^{-2}$ (Fig. 5). As shown in Fig. 5a, the cells containing only PEO and those with PEO + tetraglyme $+\mathrm{SiO}_{2}$ and $\mathrm{PEO}+$ polyglyme $+\mathrm{SiO}_{2}$ experienced a high degree of polarization during early stages of the tests and thus could not be cycled any further. While the cell containing PC could be cycled, its polarization increased continuously, possibly because of the repeated decomposition of the solvent ${ }^{7}$ (Fig. 5b). On the other hand, the cell with $\mathrm{DME}+\mathrm{PEO}+\mathrm{SiO}_{2}$ could be cycled a 100 times without a significant increase in polarization, as shown in Fig. $5 c$, confirming that our principles for electrolyte design were valid.

In summary, to overcome the problems of electrolyte decomposition and dendritic growth on the surfaces of Li metal anodes, we designed liquid electrolytes by performing $a b$ initio atomic and statistical simulations, investigating model electrolytes using Li symmetric cells, and performing in situ microscopy-based analyses. We found that the following rules were applicable for designing electrolytes suitable with $\mathrm{Li}$ anodes: the solvent should have a low reduction potential, the salt anions should be large in size, and the resulting electrolyte should have low viscosity. By combining a screened liquid electrolyte with a polymer electrolyte in a pressurized cell, we could 
achieve an excellent charging/discharging durability of around 100 cycles for an anode with an extremely high areal capacity of $12 \mathrm{mAh}$ $\mathrm{cm}^{-2}$. Although the practical use of this electrolyte composition might be limited owing to the low boiling point of DME (Supplementary Table S3), the results of this study clearly suggest that proper electrolyte design involving the control of electrolyte reactivity with respect to lithium, as well as the viscosity of the electrolyte and its anions' sizes, can increase the longevity of lithium anodes.

\section{Methods}

DFT calculations. The DFT/basis-set of the B3LYP/6-311 + G(d,p) level and the polarized continuum model $(\mathrm{PCM})^{36}$ for describing the solvation effect were used as implemented in the Gaussian 03 software package ${ }^{37}$. The reduction potential was calculated from the difference in the total energies of the reduced and neutral molecules, which were determined on the basis of the following reduction reaction:

$$
M(\text { solution })+e^{-} \text {(gas) } \rightarrow M^{-} \text {(solution), }
$$

where $M$ and $e$ denote a solvent molecule and an electron, respectively. To determine whether the calculation method used was valid, we compared the results of the calculations obtained using different DFT levels with those achieved experimentally for different solvent molecules (Supplementary Table S4).

The AIMD simulations were performed as implemented in the Vienna ab initio simulation package (VASP) $\operatorname{code}^{38}$. All the calculations were based on the DFT within the Perdew-Burke-Ernzerhof generalized gradient approximation (PBE-GGA). The projector augmented wave method, a supercell containing molecules with the exact number depending on the experimentally determined molar mass and density of each molecule, and a Li slab with 80 atoms were used. In all the calculations, a time step of $1 \mathrm{fs}$ and a Nose thermostat at $300 \mathrm{~K}$ were used. The interfacial energy was defined as

$$
E_{\mathrm{S}}(M-\mathrm{Li})=E_{\text {tot }}(M-\mathrm{Li})-E_{\text {tot }}(M ; \text { bulk })-E_{\text {tot }}(\mathrm{Li} ; \text { bulk }),
$$

where $E_{\text {tot }}(M-\mathrm{Li})$ is the total energy of the supercell comprising the solvent molecules and the Li metal slab and $E_{\mathrm{tot}}(M$;bulk) is the total energy of the bulk solvent molecules, both at $300 \mathrm{~K}$ and 1 ps. $E_{\text {tot }}(\mathrm{Li} ;$ bulk $)$ is the total energy of bulk Li.

Diffusion-limited aggregation model. A statistical model of diffusion-limited aggregation (DLA) was used. In this model, the Li ions diffused until they collided and were then attached to whatever they hit ${ }^{26}$. To describe the two-dimension-like in situ cell used, we employed a two-dimensional DLA model in which the box size was 200 $\times 6000 ; 5000$ particles were used. The bottom line of the box represented an anode, i.e., the diffused $\mathrm{Li}$ ions were caught at the line. It was assumed that the mean collision time of the Li ions was proportional to the number of grids traversed in each random diffusion ste ${ }^{28}$. To describe the drift motion, the weight probability in the direction of the anode was set higher.

Design of Li symmetric cells. Li symmetric coin cells were designed to evaluate the effect of the electrolyte on the growth of lithium dendrites, as shown in Fig. 2a. Two lithium foils (diameter: $16 \mathrm{~mm}$; thickness: $500 \mu \mathrm{m}$ ) were used as the electrodes. A polyethylene terephthalate film (diameter: $19 \mathrm{~mm}$; thickness: $100 \mu \mathrm{m}$ ), with a 12$\mathrm{mm}$ hole at its center, and a polypropylene spacer (diameter: $19 \mathrm{~mm}$; thickness: $1 \mathrm{~mm}$ ), with a $1-\mathrm{mm}$ hole at its center, were used to separate the electrodes and expose their controlled areas to improve the reproducibility of the short-circuit time $t_{\mathrm{s}}$. Finally, the electrolyte was filled into the cell and a spring, a spacer, and a gasket were installed. The Li symmetric cell was discharged (lithium stripping from the surface of the negative lithium electrode, thus cleaning it electrochemically) at a current density of $0.05 \mathrm{~mA} \mathrm{~cm} \mathrm{~cm}^{-2}$ for $10 \mathrm{~h}$ for conditioning. It was then charged (lithium deposition on the negative electrode) at $1.0 \mathrm{~mA} \mathrm{~cm}{ }^{-2}$ by means of a battery-testing system (TOSCAT-3100, Toyo System). The viscosities and conductivities of the various electrolytes were measured using a rheometer (MCR 302, Anton Paar) and a conductivity meter $\left(856\right.$ Conductivity Module, Metrohm), respectively, at $21^{\circ} \mathrm{C}$. We performed each experiment more than five times and obtained errors within $10 \%$.

Design of the in situ Li symmetric cell. In order to directly observe and record the growth of lithium dendrites in situ using a microscopy system (Axis Pro, Micro Support), an in situ observation cell was designed, as shown in Supplementary Fig. S3. Two lithium foils were used for the positive and negative electrodes. Lithium was deposited on the negative electrode at a current density of $5.0 \mathrm{~mA} \mathrm{~cm}^{-2}$ by means of a potentiostat/galvanostat (1480 Multistat, Solartron Analytical). During lithium deposition, images of the lithium dendrites were taken by the microscope at a capacity of $1.67 \mathrm{mAh} \mathrm{cm}^{-2}$; the images are shown in Fig. 3 .

Preparation of the composite electrolyte and the cycle-life tests. PEO (molecular weight: $6 \times 10^{5}$, Sigma-Aldrich;) powder, the salt LiTFSI (Wako Chemical), and nano- $\mathrm{SiO}_{2}$ (Sigma-Aldrich, 10-20 nm, Sigma-Aldrich) were dissolved in acetonitrile and stirred at room temperature for $24 \mathrm{~h}$. The weight ratio of $\mathrm{PEO} / \mathrm{LiTFSI} / \mathrm{SiO}_{2}$ was $14.7: 5.3: 1$. To synthesize polymer electrolytes, the formed slurry was cast on a Teflon dish for $24 \mathrm{~h}$ to allow the acetonitrile to evaporate and then dried at $80^{\circ} \mathrm{C}$ over a period of $24 \mathrm{~h}$ in a vacuum oven. The Li symmetric coin type cells were assembled using a liquid electrolyte (1 M LiTFSI in various solvents) and a polymer electrolyte. The cell structure was as follows: $\mathrm{Li}(0.5 \mathrm{~mm}$ thick $) /$ liquid electrolyte $(100 \mu \mathrm{L})+$ polymer electrolyte $(50 \mu \mathrm{m}) /$ separator $\left(\right.$ Celgard $\left.{ }^{\circledR} 3501\right) /$ liquid electrolyte $(100 \mu \mathrm{L})+$ polymer/Li $(0.5 \mathrm{~mm})$, where we used in excess of $200 \mu \mathrm{L}$ of electrolytes since extra electrolytes were necessary for filling the dead volume of the coin cell and squeezing out during assembly. The cycle-life measurements were performed at a current density of $3 \mathrm{~mA} \mathrm{~cm}^{-2}$ for $4 \mathrm{~h}$ for each discharge and charge step using a battery testing system (TOSCAT-3100, Toyo System). We confirmed the reproducibility of the experiments in Fig. 5, where the experimental results in Fig. 5a showed almost no difference, the ones in Fig. $5 \mathrm{~b}$ showed a range between 17 and 24 cycles, and the ones in Fig. $5 \mathrm{c}$ showed more than 100 cycles in the repeated experiments.

1. Croce, F., Appetecchi, G. B., Persi, L. \& Scrosati, B. Nanocomposite polymer electrolytes for lithium batteries. Nature 394, 456-458 (1998).

2. Tarascon, J.-M. \& Armand, M. Issues and challenges facing rechargeable lithium batteries. Nature 414, 359-367 (2001)

3. Bruce, P. G., Freunberger, S. A., Hardwick, L. J. \& Tarascon, J.-M. Li- $\mathrm{O}_{2}$ and Li-S batteries with high energy storage. Nature Mater. 11, 19-29 (2012).

4. Ji, X., Lee, K. T. \& Nazar, L. F. A highly ordered nanostructured carbon-sulphur cathode for lithium-sulphur batteries. Nature Mater. 8, 500-506 (2009).

5. Jung, H.-G., Hassoun, J., Park, J.-B., Sun, Y.-K. \& Scrosati, B. An improved highperformance lithium-air battery. Nature Chem. 4, 579-585 (2012).

6. Peng, Z., Freunberger, S. A., Chen, Y. \& Bruce, P. G. A reversible and higher-rate Li- $\mathrm{O}_{2}$ battery. Science 337, 563-566 (2012).

7. Winter, M. et al. Studies on the anode/electrolyte interface in lithium ion batteries. Monatsh. Chem. 132, 473-486 (2001).

8. Aurbach, D. et al. Recent studies of the lithium-liquid electrolyte interface electrochemical, morphological and spectral studies of a few important systems. J. Power Sources 54, 76-84 (1995).

9. Aurbach, D., Zinigrad, E., Teller, H. \& Dan, P. Factors which limit the cycle life of rechargeable lithium (metal) batteries. J. Electrochem. Soc. 147, 1274-1279 (2000).

10. Howlett, P. C., MacFarlane, D. R. \& Hollenkamp, A. F. High lithium metal cycling efficiency in a room-temperature ionic liquid. Electrochem. Solid-State Lett. 7, A97-A101 (2004).

11. Jeong, S. et al. Suppression of dendritic lithium formation by using concentrated electrolyte solutions. Electrochem. Commun. 10, 635-638 (2008).

12. Fringant, C., Tranchant, A. \& Messina, R. Behavior of lithium-electrolyte interface during cycling in some ether-carbonate and carbonate mixtures. Electrochim. Acta 40, 513-523 (1995)

13. Hirai, T., Yoshimatsu, I. \& Yamaki, J. Influence of electrolyte on lithium cycling efficiency with pressurized electrode stack. J. Electrochem. Soc. 141, 611-613 (1994).

14. Eweka, E., Owen, J. R. \& Ritchie, A. Electrolytes and additives for high efficiency lithium cycling. J. Power Sources 65, 247-251 (1997).

15. Umeda, G. A. et al. Protection of lithium metal surfaces using tetraethoxysilane. J. Mater. Chem. 21, 1593-1599 (2011).

16. Ota, H., Wang, X. \& Yasukawa, E. Characterization of lithium electrode in lithium imides/ethylene carbonate, and cyclic ether electrolytes. J. Electrochem. Soc. 151, A427-A436 (2004).

17. Suo, L., Hu, Y.-S., Li, H., Armand, M. \& Chen, L. A new class of solvent-in-salt electrolyte for high-energy rechargeable metallic lithium batteries. Nature Commun. 4, 1481 (2013).

18. Wilkinson, D. P., Blom, H., Brandt, K. \& Wainwright, D. Effects of physical constraints on Li cyclability. J. Power Sources 36, 517-527 (1991).

19. Lee, S.-H., Liu, P. \& Tracy, C. E. Lithium thin-film battery with a reversed structural configuration $\mathrm{SS} / \mathrm{Li} / \mathrm{Lipon} / \mathrm{Li}_{\mathrm{x}} \mathrm{V}_{2} \mathrm{O}_{5} / \mathrm{Cu}$. Electrochem. Solid-State Lett. 6, A275-A277 (2003).

20. Bouchet, R. et al. Single-ion BAB triblock copolymers as highly efficient electrolytes for lithium-metal batteries. Nature Mater. 12, 452-457 (2013).

21. Blomgren, G. E. Chapter 2. Physical and chemical properties of nonaqueous electrolyte solutions. Nonaqueous Electrochemistry Aurbach, D. (ed.) 56-58 (Marcel Dekker, Inc., 1999).

22. Yoshida, K. et al. Oxidative-stability enhancement and charge transport mechanism in glyme-lithium salt equimolar complexes. J. Am. Chem. Soc. 133 , 13121-13129 (2011)

23. Park, M. S. \& Doo, S. Topmost atomistic Li structures and native point defects in the $\operatorname{Li}(001)$ surface. ECS Trans. 35, 14, 1-6 (2011).

24. Monroe, C. \& Newman, J. Dendrite growth in lithium/polymer systems. J. Electrochem. Soc. 150, A1377-A1384 (2003).

25. Bard, A. J. \& Faulkner, L. R. Chapter 2. Potentials and thermodynamics of cells. Electrochemical Methods: Fundamentals and Applications 65-69 (Wiley, 2000).

26. Lin, R. et al. Microelectrode study of pore size, ion size, and solvent effects on the charge/discharge behavior of microporous carbons for electrical double-layer capacitors. J. Electrochem. Soc. 156, A7-A12 (2009).

27. Ue, M. Mobility and ionic association of lithium and quaternary ammonium salts in propylene carbonate and $\gamma$-butyrolactone. J. Electrochem. Soc. 141, 3336-3342 (1994).

28. Chaikin, P. M. \& Lubensky, T. C. Chapter 2. Structure and scattering. Principles of Condensed Matter Physics 90-97 (Cambridge Univ. Press, 1995).

29. Uwaha, M. \& Saito, Y. Aggregation growth in a gas of finite density: velocity selection via fractal dimension of diffusion-limited aggregation. Phys. Rev. A $\mathbf{4 0}$, 4716-4723 (1989). 
30. Bockris, J. O'M. \& Reddy, A. K. N. Chapter 4. Ion transport in solutions, Modern Electrochemisty: Ionics 442-456 (Plenum Press, New York and London, 1998).

31. Liu, Y., Lee, J. Y. \& Hong, L. In situ preparation of poly(ethylene oxide)- $\mathrm{SiO}_{2}$ composite polymer electrolytes. J. Power Sources 129, 303-311 (2004).

32. Scrosati, B., Croce, F. \& Perci, L. Impedance spectroscopy study of PEO-based nanocomposite polymer electrolytes. J. Electrochem. Soc. 147, 1718-1721 (2000).

33. Capiglia, C., Mustarelli, P., Quartarone, E., Tomasi, C. \& Magistris, A. Effects of nanoscale $\mathrm{SiO}_{2}$ on the thermal and transport properties of solvent-free, poly(ethylene oxide)(PEO)-based polymer electrolytes. Solid State Ionics 118, 73-79 (1999).

34. Liu, S. et al. Effect of nano-silica filler in polymer electrolyte on Li dendrite formation in $\mathrm{Li} /$ poly(ethylene oxide) $-\mathrm{Li}\left(\mathrm{CF}_{3} \mathrm{SO}_{2}\right)_{2} \mathrm{~N} / \mathrm{Li}$. J. Power Sources 195, 6847-6853 (2010)

35. Giannelis, E. P. Polymer layered silicate nanocomposites. Adv. Mater. 8, 29-35 (1996)

36. Tomasi, J., Mennucci, B. \& Cammi, R. Quantum mechanical continuum solvation models. Chem. Rev. 105, 2999-3093 (2005).

37. Frisch, M. J. et al. Gaussian-03, Revision D.02, Gaussian, Inc., Wallingford CT, 2004.

38. Kresse, G. \& Furthmüller, J. Efficient iterative schemes for ab initio total-energy calculations using a plane-wave basis set. Phys. Rev. B 54, 11169-11186 (1996).

\section{Author contributions}

M.S.P. performed the simulations. S.B.M. and D.J.L. conducted the experiments. M.S.P., S.B.M., S.D. and D.I. contributed extensively to the planning of and discussions on this work. O.Y. discussed the results and commented on the manuscript. M.S.P., S.B.M., D.J.L. and D.I. wrote the paper.

\section{Additional information}

Supplementary information accompanies this paper at http://www.nature.com/ scientificreports

Competing financial interests: The authors declare no competing financial interests.

How to cite this article: Park, M.S. et al. A Highly Reversible Lithium Metal Anode. Sci. Rep. 4, 3815; DOI:10.1038/srep03815 (2014)

cc) (i) $\odot$ This work is licensed under a Creative Commons Attribution-

BY NC ND NonCommercial-NoDerivs 3.0 Unported license. To view a copy of this license, visit http://creativecommons.org/licenses/by-nc-nd/3.0 